LA-UR- $01-2293$

Approved for public release; distribution is unlimited.

\section{\begin{tabular}{c|l} 
Title: & $\begin{array}{l}\text { COMPLEXITY AND APPROXIMABILITY OF QUANTIFIED AND } \\
\text { STOCHASTIC CONSTRAINT SATISFACTION PROBLEMS }\end{array}$
\end{tabular}}

Author(s):

Submitted to:

Harry B. Hunt, III, D-2

Richard E. Stearns, State University of NY, Albany

Madhav V. Marathe, D-2

SAT 2001: Workshop on Theory \& Applications of Satisfiability Testing Boston, Massachusetts

June 14-15, 2001

\title{
Los Alamos \\ NATIONAL LABORATORY
}

Los Alamos National Laboratory, an affirmative action/equal opportunity employer, js operated by the University of California for the U.S. Department of Energy under contract W-7405-ENG-36. By acceptance of this $\equiv$ le, the publisher recogrizes that the U.S Govemment retains a nonexclusive, royalty -free license to publish or reproduce the shed form of this contribution, or to allow others to do so, for U.S. Government purposes. Los Alamos National Laboratory reyussts that the publisher identify this article as work performed under the auspices of the U.S. Department of Energy. The Los Alamos National Laboratory strongly supports academic freedom and a researcher's right to publish; as an institution, however, the Laboratory does not endorse the viewpoint of a publication or guarantee its technical correctness. 


\title{
Complexity and Approximability of Quantified and Stochastic Constraint Satisfaction Problems
}

\author{
HARRY B. HUNT III ${ }^{2,3}$ \\ RICHARD E. STEARNS ${ }^{2}$ \\ MADHAV V. MARATHE ${ }^{1}$
}

April 25, 2001

\begin{abstract}
Let $D$ be an arbitrary (not necessarily finite) nonempty set, let $C$ be a finite set of constant symbols denoting arbitrary elements of $D$, and let $\mathbf{S}$ be an arbitrary finite set of finite-arity relations on $D$. We denote the problem of determining the satisfiability of finite conjunctions of relations in $\mathbf{S}$ applied to variables (to variables and symbols in $C$ ) by $\operatorname{SAT}(\mathbf{S})$ (by $\operatorname{SAT}_{C}(\mathbf{S})$.) Here, we study simultaneously the complexity of and the existence of efficient approximation algorithms for a number of variants of the problems $\operatorname{SAT}(\mathbf{S})$ and $\operatorname{SAT}_{C}(\mathbf{S})$, and for many different $D, C$, and $\mathbf{S}$. These problem variants include decision and optimization problems, for formulas, quantified formulas stochastically-quantified formulas. We denote these problems by Q-SAT(S), MAX-Q-SAT(S), S-SAT(S), MAX-S-SAT(S) MAX-NSFQ-SAT(S) and MAX-NSF-S-SAT(S).

The main contribution is the development of a unified predictive theory for characterizing the the complexity of these problems. Our unified approach is based on the following basic two basic concepts: (i) strongly-local replacements/reductions and (ii) relational/algebraic representability. Let $k \geq 2$. Let $\mathbf{S}$ be a finite set of finite-arity relations on $\Sigma_{k}$ with the following condition on $\mathbf{S}$ : All finite arity relations on $\Sigma_{k}$ can be represented as finite existentially-quantified conjunctions of relations in $\mathbf{S}$ applied to variables (to variables and constant symbols in $C$ ). Then we prove the following new results.
\end{abstract}

1. The problems SAT(S) and $\mathrm{SAT}_{C}(\mathbf{S})$ are both NQL-complete and $\leq_{l o g n}^{b w}$-complete for NP.

2. The problems Q-SAT(S), Q-SAT $C(\mathbf{S})$, are PSPACE-complete. Letting $k=2$, the problem S$\mathrm{SAT}(\mathbf{S})$ and $\mathrm{S}_{-} \mathrm{SAT}_{C}(\mathbf{S})$ are PSPACE-complete.

3. $\exists \epsilon>0$ for which approximating the problems MAX-Q-SAT(S) within $\epsilon$ times optimum is PSPACE-hard. Letting $k=2, \exists \epsilon>0$ for which approximating the problems MAX-S-SAT(S) within $\epsilon$ times optimum is PSPACE-hard.

4. $\forall \epsilon>0$ the problems MAX-NSF-Q-SAT(S) and MAX-NSF-S-SAT(S), are PSPACE-hard to approximate within a factor of $n^{\epsilon}$ times optimum.

These results significantly extend the earlier results by (i) Papadimitriou [Pa85] on complexity of stochastic satisfiability, (ii) Condon, Feigenbaum, Lund and Shor [CF+93, CF+94] by identifying natural classes of PSPACE-hard optimization problems with provably PSPACE-hard $\epsilon$-approximation problems. Moreover, most of our results hold not just for Boolean relations: most previous results were done only in the context of Boolean domains. The results also constitute as a significant step towards obtaining a dichotomy theorems for the problems MAX-S-SAT(S) and MAX-Q-SAT(S); a research area of recent interest [CF+93, CF+94, Cr95, KSW97, LMP99].

\footnotetext{
'Corresponding Author. Los Alamos National Laboratory, P. O. Box 1663, MS M997, Los Alamos NM 87545. The work is supported by the Department of Energy under Contract W-7405-ENG-36. Email: marathe@1anl.gov.

${ }^{2}$ Department of Computer Science, University at Albany - State University of New York, Albany, NY 12222. Research supported in part by NSF Grant CCR94-06611. Email: hunt, res@Cs . albany. edu.

${ }^{3}$ Some of the work done while author was visiting Los Alamos National Laboratory and supported by the Department of Energy under Contract W-7405-ENG-36.
} 


\section{Introduction and motivation}

Over the past thirty years, researchers in theoretical computer science, AI, operations research, combinatorial optimization, and algorithmic algebra have studied variants of satisfiability and constraint satisfaction problems. This research was motivated by the facts that (i) such problems have wide ranging applicability in modeling real life problem and (ii) these problems are also fundamental from a complexity theory stand point providing prototypical complete problems for various complexity classes. Specific research topics areas studied in these application contexts include:

1. complexity of $3 \mathrm{SAT}$ and in general Boolean constraint satisfaction problems studied in [FV93, CJ+00, JCG971

2. dichotomy type results for decision and optimization versions of Boolean constraint satisfaction problems [Cr95, KSW97, LMP99, MH+94],

3. recent research on the complexity and (non)-approximability of PSPACE-hard quantified and stochastic Boolean satisfiability problems [Pa94, Pa85, CF+93, CF+94, MH+94, HSM94, LMS96].

Here, we combine these lines of research and simultaneously study the decision, optimization and counting complexity of quantified and stochastic constraint satisfaction problems. Moreover, we do this not only for Boolean domains but for finite $(\geq 2)$ as well as infinite domains.

We use the following notation for describing constraint satisfaction problems. Throughout this paper, unless otherwise stated explicitly, $D$ is an arbitrary (not necessarily finite) nonempty set; $C$ is a finite set of constant symbols denoting elements of $D$; and $\mathbf{S}$ and $\mathbf{T}$ are (usually finite) sets of finite-arity relations/algebraic on $D$. An $\mathbf{S}$-clause (a constant free $\mathbf{S}$-clause) is a relation in $\mathbf{S}$ applied to variables and constants (to variables only respectively). An $\mathbf{S}$-clause is also sometimes referred to as a term or a constraint. An S-formula (a constant free $\mathbf{S}$-formula) is a finite nonempty conjunction of $\mathbf{S}$-clauses (constant free $\mathbf{S}$-clauses respectively). An $\mathbf{S}$-formula is satisfiable, if each individual $\mathbf{S}$-clause is simultaneously satisfiable. An S-clause $\left(S_{i}, R_{i}\right)$ with variables $S_{i}$ and the relation $R_{i}$ is satisfiable, if the assignment to the variables of the $\mathbf{S}$-clause yields a tuple that belongs to $R_{i}$.

The above definitions are given when all the variables are existential variables. We now extend the above concepts to define quantified constraint satisfaction problems. A quantified S-formula $F$ is of the form $F=$ $\left(Q_{1} x_{i_{1}}\right) \cdots\left(Q_{m} x_{i_{m}}\right) f\left(x_{1}, \ldots, x_{n}\right)$, where the following hold: (i) the variables of $F$ are $x_{i}$ for $1 \leq i \leq n$; (ii) $Q_{1}, \ldots, Q_{m} \in\{\exists, \forall\}$; and (iii) $f$ is an S-formula. We say that $F$ is constant-free, when the formula $f$ is constant-free. Let us assume that $F$ has the following structure:

$$
F=\forall x_{1} \exists x_{2}, \ldots \forall x_{n-1} \exists x_{n} F\left(x_{1}, \ldots, x_{n}\right)
$$

i.e. all odd numbered quantifiers are universal and the even numbered quantifiers are existential ${ }^{4}$. Then $F$ is satisfiable if for all values of $x_{1}$ there exists an assignment of $x_{2}$, such that for assignments to $x_{3} \ldots$ such that $F\left(x_{1}, \ldots x_{n}\right)$ evaluates to true. Alternatively, the formula $F$ is satisfiable iff there exists at least one prooftree for $F$. Papadimitriou [Pa85] introduced Stochastic Satisfiability problems. An instance of a stochastic satisfiability problem is of the form

$$
F=\mathbf{R} x_{1} \exists x_{2}, \ldots \mathbf{R} x_{n-1} \exists x_{n}\left(E\left(\boldsymbol{F}\left(x_{1}, \ldots, x_{n}\right) \geq 1 / 2\right)\right.
$$

Here $\mathbf{R}$ denotes a random quantifier. In other words, we ask if there is a random assignment to $x_{1}$ (with equiprobable values for 0 and 1) such that there exists an assignment to $x_{2}$ s.t. for a random assignment to $x_{3}$ $\ldots$ the boolean formula $F$ is satisfied with an expectation of at least a $1 / 2$. Under the notion of equiprobable values that the variables can take, this is equivalent to saying if for at least half of the leaves of a proof tree the formula evaluates to true. The universal and stochastic quantifiers can be combined the expected

\footnotetext{
${ }^{4}$ Sometimes we might have a sequence of existential or universal quantifiers.
} 
value threshold can be different from $1 / 2$ and variables need not be assigned 0 or 1 equiprobable. we will leave discussion of these extension to the full paper. See [LMP99] for details on how to incorporate these extensions as a part of a general definition.

We denote the problem of determining the satisfiability of finite conjunctions of relations in $\mathbf{S}$ or simultaneous satisfiability of systems of algebraic constraints applied to variables (applied to variables and constant

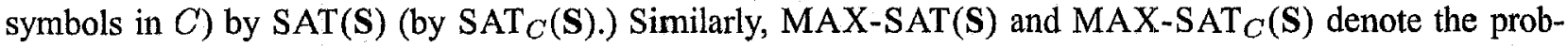
lems of satisfying maximum number of simultaneously satisfiable constant free S-clauses and S-clauses with constants.

The problem Q-SAT(S) (Q-SAT $C(\mathbf{S})$ ) is the problem of determining if a constant-free (an arbitrary) quantified S-formula is satisfiable. The problems S-SAT(S) (S-SAT $C(\mathbf{S})$ ) are similar except that now each $Q_{i} \in\{\exists, \mathbf{R}\}$. here $\mathbf{R}$ is called random quantifier. The problems MAX-Q-SAT(S), MAX-Q-SAT $C(\mathbf{S})$, MAX-S-SAT(S), MAX-S-SAT $C(S)$ are the optimization versions of these problems that aim to maximize the minimum number of simultaneously satisfiable clauses (or satisfiable with probability greater than $1 / 2$ ) over all partial proof trees.

In the second class of optimization problems, we are interested in satisfying the maximum number of formulas, when instances consist of conjunction of S-formulas. Given a set of S-formulas $\phi=\left\{\phi_{1}, \cdots, \phi_{n}\right\}$ the problem MAX-NSF-SAT(S) is to find a truth assignment to the variables satisfying the maximum number of formulas in $\phi$. Extensions to MAX-NSF-Q-SAT(S) and MAX-NSF-S-SAT(S) follow the above schemata and are omitted.

Important Note: Due to space limitations, the abstract contains only a discussion of the results, overview of techniques used and significance of the results. Full proofs and detailed definitions are given in the full paper that can be obtained from the authors. Formal definitions of these problems can be found in [CF+93, CF+94, LMP99, HSM94, LMS96].

Example 1: The generalized CNF satisfiability problems $\mathrm{SAT}(\mathrm{S})$ and $\mathrm{SAT}_{c}(\mathrm{~S})$ generalize the problems 3SAT, 1-3SAT, NAE-3SAT, etc. in [GJ79]. For example, let $E O(x, y, z)$ be the ternary logical relation given by $\{(1,0,0),(0,1,0),(0,0,1)\}$. Then, the problem EXACTLY-1-IN-EX3-MONO-SAT is the same as the problem $\operatorname{SAT}(\{\mathrm{EO}\})$. An instance of the above problem might consist of the set of variables $x, \boldsymbol{y}, z, w$ and the formula $F=E O(x, y, z) \wedge E O(x, y, w) \wedge E O(x, w, z)$. It is easy to see that $F$ is satisfiable by setting $x=1$ and setting all other variables to 0 . Let $f$ be an S-formula with $m$ clauses and $n_{i}$ literals in clause $i, 1 \leq i \leq m$. The size of $f$ denoted by size $(f)$ is given by $O\left(\sum_{i=1}^{i=m} n_{i}\right)$. Let $C$ be a set of cllauses defined over a set of variables $V$. We will use $F(C, V)$ to denote the formula obtained by the conjunction of clauses in $C$. By appropriately defining unary, binary and ternary versions of the relation $E O$, it is possible to define 1-3-SAT problem.

Our general results in many cases do not depend on the domain being binary or even finite. Similarly, it is possible to have finite or infinite constraint relations. This happens naturally, when we deal with algebraic constraints where constraints can be specified using algebraic (in)equations.

Example 2: Extensions of constraint satisfaction problems to quantified and stochastically quantified constraint satisfaction problems is done by allowing one to use first order quantifiers. Consider again the $E O$ relation as defined in Example 1. An instance $I$ of Q-SAT $(E O)$ might look like $\forall x \exists y \forall z \exists w F$, where $F$ is as defined above. Then it can be verified that $I$ is not satisfiable. Moreover, $\mathbf{R} x \exists y \mathbf{R} z \exists w \boldsymbol{F}$ is also not satisfiable. On the other hand $\forall x \exists y \forall z \exists w_{1} \forall q \exists w_{2}\left(\left(E O\left(x, y, w_{1}\right) \wedge E O\left(z, y, w_{2}\right)\right)\right.$ is satisfiable. 


\section{Summary of results}

As mentioned earlier, the focus of this paper is to derive unified technique for characterizing the computational complexity and efficient approximability of quantified and stochastic satisfiability problems. For most part, we concentrate on the quantified and stochastic versions of the problems; the results for unquantified versions are derived in-situ. Specific results obtained in this paper are summarized in Figure 1. The general contributions of this paper include the following.

(1) We formalize an infinite class of quantified and stochastic constraint satisfaction problems. The type of problems studied include: decision, counting and optimization versions of these problems. Furthermore, combining these with the recent ideas of Littman et. al. we can define more general variants of the problem in which we vary the quantifiers and their semantics. We suspect that these infinite classes of problems will play a role similar to that already played by their unquantified counterparts in the earlier development of complexity theory. Of special note is the formalism of optimization and counting versions of these problems: these problems have not be been defined and studied in the literature prior to this paper. Recently there has been interest in studying the approximability of PSPACE-hard optimization problems:

(2) We formalize two simple yet important concepts: local replacements/reductions and relational representability. We derive the basic complexity theoretic properties related to these concepts. Using these concepts, we propose unified methods for characterizing simultaneously, the decision, optimization, approximate optimization and counting complexity of quantified and stochastic constraint satisfaction problems.

(3) We derive very general sufficient conditions and generic reductions that simultaneously show that the decision and the approximate optimization problems are hard for their respective complexity classes. There has been a recent interest in studying the approximability of PSPACE-hard optimization problems. Our general results yield an infinite set of maximization versions of stochastic and quantified constrained satisfaction problems that are PSPACE-hard to approximate beyond a certain fixed constant and another infinite set that are PSPACE-hard to approximate for any $n^{\epsilon}, \epsilon>0$. Since the influential paper by Papadimitriou and Yannakakis on MAX SNP, there has been interest in finding logical/algebraic characterization of NP-hard optimization problems that are hard to approximate within different factors. The results for MAX-Q-SAT(S), MAX-SSAT(S) MAX-NSF-Q-SAT(S) and MAX-NSF-S-SAT(S) provide similar algebraic characterizations of quantified and stochastic PSPACE-hard optimization problems.

We now discuss some of the specific results obtained in this paper and simultaneously contrast them with known results from the literature. These results are summarized in Figure 1. In order to allow for an easy comparison between the results obtained here and the results obtained earlier by other researchers, we summarize both the results in the Figure. Moreover, previous results and our results are in 1-1 correspondence in terms of the numbering used. So for instance, 3(b) in Part 1, summarizes the earlier result on non-approximability of MAX-Q-3SAT, our result is given as 3(b) in Part 2.

Much of this discussion, but by no means all, is limited to finite sets $D$, since all hardness results given here are tight when $D$ is finite. Almost all resulting reductions are local. Thus, they are $O(n \cdot \log n)$ time-, linear size-, and $O(\log n)$ space-bounded.

\subsection{Discussion and Significance}

We discuss some of the above specific results in some detail. Note that some of the results that follow as corollaries of our general theorems have also been obtained previously by us or other researchers. Our purpose here is to demonstrate the effectiveness of the unified approach and to show that general results presented contain much of the earlier results as subsets of the general results. Moreover, the unified approach yields a large collection of new results that are reported for the first time in the literature. We make the 


\section{Part 1: Summary of related results applicable to this paper}

1. [Sc78, CES85, MS81]:The problems 3SAT and 3-COLORABLE GRAPH are NQL-complete. The problems EX-3SAT, EXACTLY 1-EX3MONOSAT, NAE-EX3SAT, GOLD'S-MONOTONE-3SAT are $\leq_{\text {logn }}^{b w}$-complete for NP.

2. [Sc78, Pa85]:The problems $\operatorname{SAT}(\mathbf{S})$ and $\operatorname{SAT}_{C}(\mathbf{S})$ are $\mathbf{N P}$-complete and the problems Q-SAT(S) and Q-SAT $_{C}(\mathbf{S})$ are PSPACE-complete, for all finite sets $\mathbf{S}$ of finite-arity Boolean relations such that $\operatorname{Rep}_{C}(\mathbf{S})=$ BOOLEAN-RELATIONS ${ }^{a}$. The problem S-3SAT is PSPACE-complete.

3. (a) [ALM+98, PY91]: The problems MAX-3SAT and MAX-NAE-3SAT are MAX SNP-complete. Consequently, there exists $\epsilon>0$ for which approximating these two problems within $\epsilon$ times optimum is NP-hard.

(b) $[\mathrm{CF}+93]$ : $\exists \epsilon>0$ for which approximating the problem MAX-Q-3SAT within $\epsilon$ times optimum is PSPACE-hard.

(c) [CF+94]: $\exists \epsilon>0$ for which approximating the problem MAX-S-3SAT within $\epsilon$ times optimum is PSPACE-hard.

4. (a) [PR93]:The problem MAX-NSF-3SAT is MAX $\Pi_{1}$-complete. Consequently for all $\epsilon>0$ approximating this problem within $\epsilon$ times optimum is NP-hard.

(b) $[\mathrm{CF}+93]$ :For all $\epsilon>0$ approximating the problem MAX-Q-FoRMULA-3SAT within $\epsilon$ times optimum is PSPACE-hard.

\section{Part 2: Summary of the results obtained in this paper}

Let $k \geq 2$. Let $\mathbf{S}$ be a finite set of finite-arity relations on $\Sigma_{k}$ such that $\operatorname{Rep}(\mathbf{S})=\boldsymbol{\Sigma}_{\mathbf{k}}-\mathbf{R E L A T I O N S} .^{b}$ Then the following hold:

1. The problems SAT(S) and SAT $C(\mathbf{S})$ are both NQL-complete and $\leq_{\log n}^{b w}$-complete for NP.

2. The problems Q-SAT(S), Q-SAT $C(\mathbf{S})$, are PSPACE-complete. Letting $k=2$, the problem $\mathrm{S}-\mathrm{SAT}(\mathbf{S})$ and S-SAT $C(\mathbf{S})$ are PSPACE-complete.

3. Let $k \geq 2$. Let $\mathbf{S}$ be any finite set of finite-arity relations on $\Sigma_{k}$ such that $\operatorname{Rep}(\mathbf{S})=\Sigma_{k}$-RELATIONS. Then, the following hold:

(a) The problem MAX-SAT(S) is MAX SNP-complete. Consequently, there exists $\epsilon>0$ such that approximating the problem within $\epsilon$ times optimum is $\mathbf{N P}$-hard.

(b) $\exists \epsilon>0$ for which approximating the problems MAX-Q-SAT(S) within $\epsilon$ times optimum is PSPACEhard.

(c) Letting $k=2, \exists \epsilon>0$ for which approximating the problems MAX-S-SAT(S) within $\epsilon$ times optimum is PSPACE-hard.

4. Let $S$ and $T$ be finite sets of finite-arity relations on an arbitrary nonempty set $D$. Let $\epsilon>0$. Then, the following hold:

(a) The problem SAT(S) is $O(n \cdot \log n)$ time-, linear size-, and $O(\log n)$ space-bounded reducible to the problem of approximating the problem MAX-NSF-SAT(S) within a factor of $\epsilon$ times optimum. Therefore whenever the problem SAT(S) is NP-hard, approximating the problem MAX-NSF-SAT(S) within $\epsilon$ times optimum is NP-hard.

(b) The problems Q-SAT(S), Q-SAT(S) are $O(n \cdot \log n)$ time-, linear size-, and $O(\log n)$ space-bounded reducible to the problems of approximating the problems MAX-NSF-Q-SAT(S), MAX-NSF-S-SAT(S), respectively, within a factor of $n^{\epsilon}$ times optimum.

${ }^{a}$ This is the terminology used in [Sc78] to say that we can represent all finite arity-boolean relations.

${ }^{h}$ Like in Boolean case, this means that all finite arity relations on $\Sigma_{k}$ can be equivalently represented as finite existentiallyquantified conjunctions of relations in $\mathbf{S}$ applied to variables (to variables and constant symbols in $C$ ).

Figure 1: Summary of results for constrained satisfaction problems. Note that a few of the results have been obtained earlier. The purpose here is to show the unified use of our techniques. 
following additional observations about the results summarized above.

First, note that several simple but fundamental properties of our model, that generalize those of previous models such as the generalized CNF satisfiability problems, the constrained satisfiability problems, and the classes of graphical problems $E C C$ and $L C C$ of [Sc78, FV93, JCG97, CJ+00, JCG97], respectively.

1. Most of our constructions hold, for domains $D$ of arbitrary not necessarily finite cardinality. Moreover, they hold for problems expressed in terms of fairly arbitrary sets of algebraically-expressed constraints $\mathbf{S}$ on $D$. In particular, these sets of constraints also need not be finite.

2. Most of our constructions use the Boolean operator and, only in the sense of simultaneously satisfiable over the domain $D$ and given set of constraints from $\mathbf{S}$.

3. All of our constructions are explicitly expressed as strongly-local graph /hypergraph replacements. This guarantees their extensibility.

Second, the problems MAX-Q-SAT(S) and MAX-S-SAT(S) are PSPACE-hard (as opposed to NPhard) to approximate beyond a fixed constant (a separate constant for each problem). Moreover MAX-NSFQ-SAT(S) and MAX-NSF-S-SAT(S) are PSPACE-hard within any $n^{\epsilon}$ factor. Thus our results provide natural algebraic classes of optimization problems that can be potentially used for proving non-approximability of PSPACE-hard optimization problems. The un-quantified version of these problems have been used in the past to derive a number of non-approximability results. Similar results can be now obtained in a game theoretic setting. For e.g. it is possible to define a game theoretic version of the MAX-CUT problem: our results how that it is PSPACE-hard to approximate.

Third, except for results in [FV93, JCG97] on when the problems SAT(S) are polynomially solvable and the well-known results that, the problems $k$-COLORABLE-GRAPH and MAX- $k$-COLORABLE-GRAPH are NP- and MAX SNP-complete, respectively, very few general hardness results were known previously for sets of relations on sets $D$ such that $3 \leq|D|<\infty$.

Our results extend earlier results and/or answer open problems in (i)Condon, Feigenbaum, Lund and Shor [CF+93, CF+94] to identify natural classes of PSPACE-hard optimization problems with provably PSPACE-hard $\epsilon$-approximation problems, (ii) work of Papadimitriou [Pa85] on stochastic satisfiability problems (where only S-3SAT was considered) and (iii) Schaefer [Sc78] on quantified generalized satisfiability problems extending it to non-Boolean domain and providing tighter reductions). Progress is made on the approximability of the problems MAX-S-SAT(S) and MAX-Q-SAT(S): a significant step towards obtaining a dichotomy theorems for these problems. recently there has been substantial interest in obtaining dichotomy results for decision, optimization and counting versions of satisfiability problems. [CF+93, CF+94, Cr95, KSW97, LMP99]. While (non)-approximability of NP-hard optimization problems has received a lot of attention over the recent years, approximability of PSPACE-hard optimization problems has only been studied by us [HSM94, MH+94] for quantified and succinctly specified problems, by Condon, Feigenbaum, Lund and Shor [CF+93, CF+94] for quantified and stochastic satisfiability problems and by Lincoln, Mitchell and Scederov in the context of linear logic [LMS96].

\section{Overall technique}

Our methodology is based upon the following two simple yet powerful concepts.

1. Relational Representability: As the name suggests, letting $\mathbf{S}$ and $\mathbf{T}$ be sets of relations/algebraic constraints on a common domain $D$, the intuitive concept that the relations in $\mathbf{S}$ are expressible (or extending the terminology from [Sc78] are representable) by finite conjunctions of the relations in $\mathbf{T}$. This is formalized in Definition 3.1 below:

Definition 3.1 1. We denote the set of all finite-arity relations on a non-empty set $D$ logically equivalent to finite existentially-quantified conjunctions of relations/algebraic constraints in $\mathbf{S}$ applied to variables 
(to variables and constant symbols in $C$ ) by $\operatorname{Rep}(\mathbf{S})($ by $\operatorname{Rep} C(\mathbf{S})$.)

2. We say that a relation $S$ is, and a set of relations $\mathbf{S}$ are, representable (constant-free representable) by $a$ set of relations/algebraic constraints $\mathbf{T}$ if and only if $S \in \operatorname{Rep}_{C}(\mathbf{T})(S \in \operatorname{Rep}(\mathbf{T}))$ and $\mathbf{S} \subset \operatorname{Rep}{ }_{C}(\mathbf{T})$ ( $\mathbf{S} \subset \operatorname{Rep}(\mathbf{T})$, respectively.)

Note: Throughout this paper $\operatorname{Rep}(\mathbf{S})$ denotes the set of relations expressible by constant-free $\mathbf{S}-f o r m u l a s ;$ and $\operatorname{Rep}_{C}(\mathbf{S})$ denotes the set of relations expressible by $\mathbf{S}$-formulas with constants from $C$.

Variants of the concepts of Definition 3.1 on the relative representability of ordered-pairs $(\mathbf{S}, \mathbf{T})$ of sets of relations, henceforth denoted collectively by relational representability, are well known, especially in mathematical logic. Previously in complexity theory, relational representability as used here and the individual constraint satisfaction problems studied have usually been restricted to finite sets $\mathbf{S}$ of finite-arity relations on finite sets $D$, generally the set $\{0,1\}$. Additionally, their uses are generally restricted to formulas or (occasionally also to quantified formulas), [Ho97, CES85, GJ79, JCG97, Sc78]. In contrast, our results apply with the exception of the problems S-SAT(S) to both finite and infinite domains and sets of relations/constraints.

For any set $D$ and finite sets of finite-arity relations $\mathbf{S}$ and $\mathbf{T}$ on $D$, if $\mathbf{S} \subset \operatorname{Rep}(\mathbf{T})$ (or $\mathbf{S} \subset \operatorname{Rep} C(\mathbf{T})$ ), then

1. the problem SAT(S) is 1-strongly local reducible to the problem $\operatorname{SAT}(\mathbf{T})$ (or $\operatorname{SAT}_{C}(\mathrm{~T})$ ),

2. the problem Q-SAT(S) is efficiently reducible to the problem Q-SAT(T) (or Q-SAT $C(T)$ ), and

3. (when $D$ is finite) the problem S-SAT(S) is efficiently reducible to the problem S-SAT(T) (or S-SAT $C(\mathbf{T})$ ).

4. Moreover often, the reductions of items 1-3 can also be used to relate the relative complexities of the associated MAX-problems.

Figure 2: Meta-Result 2. Relational Representability and Strongly-Local Reductions.

2. Local Replacements: Let $k \geq 1$. The second basic component of our methodology consists of the formalization and systematic investigation of the properties of the classes of $k$-strongly-local and $k$-stronglylocal-enforcer replacements and reductions, especially with respect to constraint satisfaction problems. The basic idea of local reductions is not new and can be traced back to [GJ79] for decision problems, and recently in [HSM94, KSW97, Cr95] for optimization problems. The new contribution of this and companion papers is to formalize the complexity theory properties of such reductions. In contrast, previous researchers, e.g. [GJ79, CES85], have discussed efficient reductions by local replacement; but they have not gone far in formalizing, or in characterizing the complexity-theoretic properties of, their concepts.

Let $k \geq 1$. Let $D_{1}, D_{2}$ be nonempty sets. Let $\mathbf{S}$ with $|\mathbf{S}|=p$ and $\mathbf{T}$ with $|\mathbf{T}|=q$ be finite nonempty sets of finite-arity relations on $D_{1}$ and $D_{2}$, respectively. We define $k$-strongly-local and $k$-strongly-local-enforcer reductions of the problem SAT(S) to the problem SAT(T) to be $k$-strongly-local and $k$-strongly-local-enforcer replacements from the set of all S-formulas to the set of all $\mathrm{T}$-formulas, that are also reductions Intuitively, $\forall k$, in $k$-strongly-local replacements we have templates, to be treated as macros, with the same template for each variable and distinct templates for each S-clause. Details about macro expansions and the the way the variables are replaced depend very simply on the value of $k$. Figure 2 shows how local replacement/reductions and relational representability can be combined to obtain efficient reductions between classes of satisfiability problems. 


\section{References}

[ALM+98] S. Arora, C. Lund, R. Motwani, M. Sudan and M. Szegedy, "Proof Verification and Hardness of Approximation Problems", J. of the ACM (JACM), 45, 1998, pp. 501-555.

[AC+98] G. Ausiello, P. Crescenzi, G. Gambosi, V. Kann, A. Marchetti-Spaccamela and M. Protasi Complexity and Approximation: Combinatorial Optimization Problems and Their Approximability Properties, Springer Verlag, 1999.

[Bo88] H. Bodlaender, Dynamic programming on graphs of bounded treewidth. Proc. 15th International Colloquium on Automata Languages and Programming (ICALP), LNCS Vol. 317, 1988, pp. 105-118.

[CES85] M. Chung, W. Evangelist and I. Sudborough. Complete problems for space bounded subclasses of NP. Acta Informatica, 22, 1985, pp. 379-395.

[CJ+00] D. Cohen, P. Jeavons, P. Jonsson, and M. Koubarakis Building Tractable Disjunctive Constraints Journal of the $A C M(J A C M), 47$ (2000), pp. 826-853.

[CF+93] A. Condon, J. Feigenbaum, $\mathrm{C}$. Lund and $\mathrm{P}$. Shor, "Probabilistically Checkable Debate Systems and Approximation Algorithms for PSPACE-Hard Functions," in Chicago Journal of Theoretical Computer Science, Vol. 1995, No. 4.

[CF+94] A. Condon, J. Feigenbaum, C. Lund and P. Shor, "Random debaters and the hardness of approximating stochastic functions," SIAM J. Computing, 26, 1997, pp. 369-400.

[Cr95] N. Creignou, "A dichotomy theorem for maximum generalized satisfiability problems," $J$. of Computer and System Sciences (JCSS), 51, 1995, pp. 511-522.

[FV93] T. Feder and M. Vardi, "Monotone monadic SNP and constraint satisfaction,". Proc. 25th Annual ACM Symposium on the Theory of Computing (STOC), 1993, pp. 612-622.

[GJ79] M. Garey and D. Johnson, Computers and Intractability: A Guide to the Theory of NP-Completeness, W. H. Freeman, San Francisco, 1979.

[Ho97] D. Hochbaum(Ed.), Approximation Algorithms for NP-Hard Problems, PWS Publishing Company, Boston, MA, 1997.

[HSM94] H. Hunt III, R. Stearns and M. Marathe, "Generalized CNF satisfiability problems and non-efficient approximability," Proc. 9th Annual IEEE Conf. on Structure in Complexity Theory, 1994, pp.356-366.

[JCG97] P. Jeavons, D. Cohen and M. Gyssens. Closure properties of constraints. Journal of the ACM, (J. ACM) 44(4):527-548, July 1997.

[KSW97] S. Khanna, M. Sudan, D. Williamson, "A Complete classification of the approximability of maximization problems derived from Boolean constraint satisfaction," Proc. 29th Annual ACM Symposium on Theory of Computing (STOC), pp. 11-20, 1997.

[Li82] D. Lichtenstein, "Planar Formulae and their Uses", SIAM J. Computing, Vol 11, No. 2, May 1982 , pp. 329-343.

[LMS96] P.D. Lincoln, J.C. Mitchell and A. Scederov. "Optimization Complexity of Linear Logic Proof Games," Theoretical Computer Science 227 (1999) pp. 299-331.

[LMP99] M. Littman, S. Majercik and T. Pitassi "Stochastic Boolean Satisfiability," Journal of Artificial Intelligence, Kluwer Publications, 2000.

[MS81] B. Monien and I. Sudborough. Bounding the bandwidth of NP-complete problems," Theoretical Computer Science, 41(2-3): 141-167, December 1985.

[MH+94] M. Marathe, H. Hunt III, R. Stearns and V. Radhakrishnan, "Approximation Algorithms for PSPACE-Hard Hierarchically and Periodically Specified Problems," in SIAM J. Computing, 27(5), pp. 1237-1261, Oct. 1998.

[Pa85] C. Papadimitriou, "Games against nature," J. Computer and System Sciences (JCSS), 31, 1985, pp. 288-301.

[PY91] C. Papadimitriou and M. Yannakakis. Optimization, approximation, and complexity classes. J. Computer and System Sciences (JCSS), 43, 1991, pp. 425-440.

[Pa94] C. Papadimitriou. Complexity Theory, Addison-Wesley, Reading, MA, 1994.

[PR93] A. Panconesi and D. Ranjan. Quantifiers and approximations. Theoretical Computer Science (TCS), 107, 1993, pp. 145-163.

[Sc78] T. Schaefer, "The complexity of satisfiability problems," Proc. 10th Annual ACM Symposium on Theory of Computing (STOC), 1978, pp. 216-226. 